\title{
Malignant Acanthosis Nigricans: An Indicator of Internal Malignancy
}

\author{
Muhammad Rizwan1, Nadia Iftikhar1, Tariq Sarfraz² and Obed Ullah1 \\ 1Department of Dermatology, Pak Emirates Military Hospital, Rawalpindi, Pakistan \\ 2Department of Pathology, Army Medical College, Rawalpindi, Pakistan
}

\begin{abstract}
Acanthosis nigricans is a skin disease characterised by symmetric velvety hyperpigmented, verrucous plaques of the intertriginous surfaces of the axilla, neck, inframammary, and mucocutaneous regions. Malignant acanthosis nigricans is a paraneoplastic phenomenon most commonly associated with gastric adenocarcinoma. Tripe palms, florid cutaneous papillomatosis and oral involvement along with generalised acanthosis nigricans are the features which are highly suggestive of internal malignancy. This case report describes a rare case of a patient who presented with combination of malignant acanthosis nigricans, tripe palms, florid cutaneous papillomatosis and oral involvement associated with undifferentiated malignant neoplasm of hepatic bile ducts.
\end{abstract}

Key Words: Acanthosis nigricans, Florid cutaneous papillomatosis, Tripe palms.

How to cite this article: Rizwan M, Iftikhar N, Sarfraz T, Ullah O. Malignant acanthosis nigricans: an indicator of internal malignancy. J Coll Physicians Surg Pak 2019; 29(9):888-890.

\section{INTRODUCTION}

The term "Acanthosis Nigricans" (AN) is derived from Greek and Latin words (acanthos, from the Greek for "thorn" and nigricans, from the Latin meaning, "black"). ${ }^{1}$ Eight different types of acanthosis nigricans have been described in the literature which are acral, benign, malignancy-associated, medication-induced, mixed type, obesity-associated, syndromic, and unilateral. 2 But broadly, it can be divided into benign and malignant types. ${ }^{3}$ Clinically, malignant acanthosis nigricans (MAN) is indistinguishable from benign acanthosis nigricans. However, rapid onset of skin lesions, additional paraneoplastic findings, ${ }^{4}$ e.g. tripe palms, extensive involvement, lesions in atypical sites like mucous membranes, unexplained weight loss and old age suggest an underlying malignancy.

We present a case of MAN with extensive involvement, of oral mucosa, tripe palms and soles, and florid papillomatosis, occurring in association with undifferentiated malignant neoplasm of hepatic bile ducts. A thorough search of literature shows that only two associated cases of bile duct malignancy are reported worldwide; whereas, none has been reported from Pakistan. The purpose of this case report is showing rarity of this association.

\section{CASE REPORT}

A 62-year gentleman, non-diabetic and non-hypertensive, presented with 8 months' history of generalised itching,

Correspondence to: Dr. Muhammad Rizwan, Department of Dermatology, Pak Emirates Military Hospital, Rawalpindi, Pakistan

E-mail: rizwan_rawalian2000@yahoo.com

Received: December 26, 2018; Revised: March 04, 2019; Accepted: March 04, 2019 darkening of skin, and 2 months' history of thickening of palmar and plantar skin. Darkening of skin started from upper back and progressed to involve entire body with accentuation on flexural areas. Skin thickening of both palms and soles was associated with itching and pain over pressure bearing areas. He also lost $10 \mathrm{Kg}$ of weight in last 6 months. There was no associated history of night sweats, fever, difficulty in swallowing, bone pains, urinary or bowel complaints, cough, seizures and weakness of any limb.

Dermatological examination showed hyperpigmentation with multiple papular lesions on face, scalp and neck coalescing to form plaques. Fissuring at angle of mouth with velvety verrucous plaques distributed over buccal mucosa and extending over hard palate were seen (Figure 1). Verrucous thickening of vermillion zone of lips was also noted. Examination of trunk revealed generalised hyperpigmentation with multiple dark coloured papules coalescing to form velvety plaques over lower abdomen (Figure 2). There were multiple excoriation marks and linear areas of hypopigmentation over upper back. Multiple verrucous lesions were present over back of neck, trunk and lower limbs. Palms and soles showed hyperkeratotic, ridged, plaques over palms and soles, sparing central palms and instep of soles (Figures 3 and 4). Warty lesions similar to those on trunk were also noted on dorsal aspects of hands and feet. Nails were normal. Rest of the systemic examination was unremarkable.

His laboratory workup revealed normal blood complete picture, bilirubin was $30 \mu \mathrm{mol} / \mathrm{L}$, alanine amino transferase $68 \mathrm{U} / \mathrm{L}$ and alkaline phosphatase was $874 \mathrm{U} / \mathrm{L}$. Human immunodeficiency virus (HIV) and hepatitis serology were negative and carcinoembryonic antigen (CEA), alpha fetoprotein (AFP), carbohydrate antigen (CA) 19-9, 


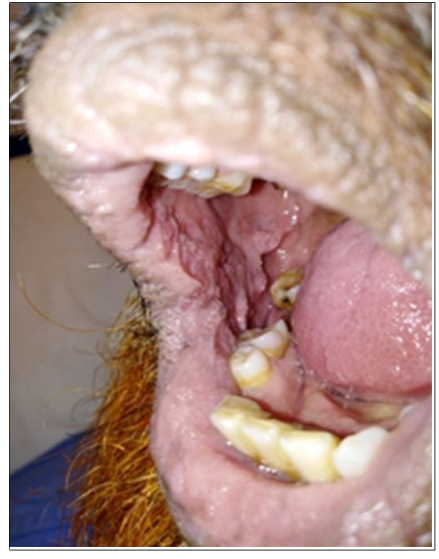

Figure 1: Velvety verrocous plaque on oral mucosa.

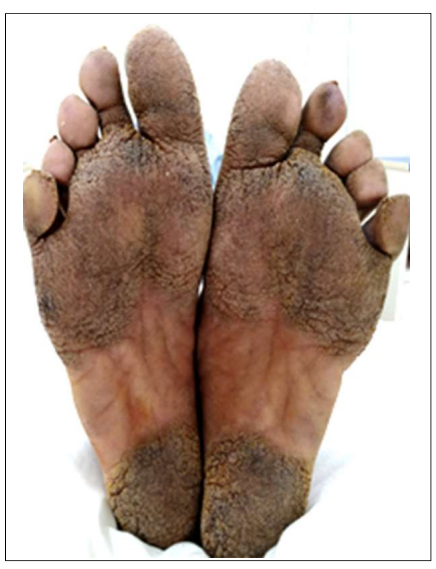

Figure 3: Hyperkeratotic plaque on soles.

and blood sugar were all normal. His ultrasound (USG) abdomen showed well defined echogenic focus measuring $1.7 \times 1.3 \mathrm{~cm}$ in proximal common bile duct (CBD), suggestive of stones. ERCP showed multiple filling defects in CBD, suggestive of stones. Multiple attempts at stone extraction were unsuccessful and a straight plastic stent was placed in CBD with free bile flow. Contrast Enhanced CT (CECT) abdomen showed and ill-defined eccentric mass lesion at the confluence of right and left hepatic ducts measuring approximately 2.9 $\times 3.2 \times 2.7 \mathrm{~cm}$ in size, with resultant upstream dilatation of intrahepatic biliary channels. CECT chest showed no evidence of metastatic disease in either lung. MR cholangio-pancreatography showed distended CBD measuring $15 \mathrm{~mm}$, with multiple signal voids suggestive of calculi and sludge balls. However, proximally close to the confluence, there was another signal void which showed internal vascularity on complementary doppler ultrasound, suggesting a mass like intraluminal Klatskin tumor rather than a sludge ball. Ultrasound-guided biopsy of the hepatic ductal mass was done and histopathology showed an undifferentiated malignant neoplasm. An extensive panel of immunostains like, Pan-CK, CK-7, CK-19, Hep Par-1, CD-34, CD-31, leucocyte common antigen (LCA), S-100, prostate specific antigen
(PSA), synaptophysin, chromogranin, CD-117, DOG-1, calretinin, and vimentin, were applied to ascertain the histogenesis of this neoplasm, but all immunostains were negative.

Patient was offered right hemihepatectomy with excision of confluence of hepatic duct and extrahepatic duct, but he declined the procedure and opted instead for palliative chemotherapy.

\section{DISCUSSION}

Acanthosis nigricans is a skin disease having symmetric velvety, verrucous, hyperpigmented plaques of the flexural areas of the axilla, neck, inframammary, and mucocutaneous regions. 5 MAN is most commonly associated with gastric adeno-carcinoma, ${ }^{6}$ but can also be associated with many other internal malignancies like pancreatic, ovarian, endometrial, cervical, breast, testicular, lung, kidney, liver, esophageal, prostatic, pharyngeal, and adenocarcinoma of the gallbladder and lymphoma. ${ }^{7}$

MAN has features like pruritis, hyperpigmented and hyperkeratotic plaques with velvety papillomas and more extensive distribution than benign acanthosis nigricans. ${ }^{8}$ Tripe palms are characterised by velvety, verrucous thickening of the palms with exaggeration of skin markings. 9 This case presents the rare association of undifferentiated malignant neoplasm of hepatic bile duct with MAN, tripe palms, oral mucosal lesions and florid cutaneous papillomatosis. Factors that stimulate epidermal keratinocytes and dermal fibroblast proliferation are the causal elements of acanthosis nigricans. In the benign form, this factor is probably insulin or an insulin-like growth factor that leads to epidermal cell proliferation. ${ }^{2}$ But in case of MAN, increased levels of transforming growth factor (TGF) secreted by tumors and stimulating epidermal growth factor receptor (EGFR) leading to epidermal hyperproliferation and differentiation, are responsilble.2,5 Other hormones like thyroid stimulating hormone, human growth hormone, melanocortin-stimulating hormone, and male sexual hormones, have been reported to play a variable etiologic role in MAN.10

Diagnosis of MAN is generally clinical. Skin biopsy is usually not required; however, it may reveal basket-weave hyperkeratosis, papillomatosis, and hyper-pigmentation of the basal layer, and some dermal papillae projecting upward in the form of finger-like projections.

In conclusion, when an atypical acanthosis nigricans is encountered, extending beyond the flexures with involvement of the oral mucosa, one needs to evaluate for an underlying malignancy. The presence of tripe palms and soles and florid cutaneous papillomatosis, augment the need to evaluate for an underlying malignancy.

\section{CONFLICT OF INTEREST:}

Authors declared no conflict of interest. 


\section{AUTHORS' CONTRIBUTION:}

MR, NI, TS, OU: Designed the case report, analysed and interpretated the data; drafted the case report and revised it for intellectual content; approved the final version to be published; agreed to be accountable for all aspects of the work.

\section{REFERENCES}

1. Rogers DL. Acanthosis nigricans. Semin Dermatol 1991; 10 : 160-3.

2. Phiske MM. An approach to acanthosis nigricans. Indian Dermatol Online J 2014; 5:239-49.

3. Cruz Jr PD, Hud Jr JA. Excess insulin binding to insulin-like growth factor receptors: proposed mechanism for acanthosis nigricans. J Invest Dermatol 1992; 98(6 Suppl):82S-85S.

4. Silva JA, Mesquita Kde C, Igreja AC, Lucas IC, Freitas AF, Oliveira SM, et al. Paraneoplastic cutaneous manifestations: concepts and updates. An Bras Dermatol 2013; 88:9-22.
5. Kutlubay Z, Engin B, Bairamov O, Tüzün Y. Acanthosis nigricans: A fold (intertriginous) dermatosis. Clin Dermatol 2015; 33:466-70.

6. Stawczyk-Macieja M, Szczerkowska-Dobosz A, Nowicki R, Majewska H, Dubowik M, Sokolowska-Wojdylo M. Malignant acanthosis nigricans, florid cutaneous papillomatosis and tripe palms syndrome associated with gastric adenocarcinoma. Postepy Dermatol Alergol 2014; 31:56-8.

7. Zhang N, Qian Y, Feng AP. Acanthosis nigricans, tripe palms, and sign of leser-Trélat in a patient with gastric adenocarcinoma: case report and literature review in China. Int $J$ Dermatol 2015; 54:338-42.

8. Singh SK, Rai T. A rare case of malignant acanthosis nigricans in a lady with ovarian cancer. Indian Dermatol Online J 2013; 4:125-7.

9. Danesh MJ, Saylor DK, Leard LE, North JP, Fox LP. Acquired acanthosis nigricans with tripe palms in a patient with interstitial lung disease. JAAD Case Reports 2016; 2:59-62.

10. Weiss $E$, Schmidberger $H$, Jany R, Hess CF, Bamberg M. Palliative radiotherapy of mucocutaneous lesions in malignant acanthosis nigricans. Acta Oncologica 1995; 34:265-7. 